\title{
A rare case presentation of acute myo-pericarditis in a patient with lupus flare
}

\begin{abstract}
Systemic lupus erythematosus (SLE) is a chronic, multisystem, inflammatory disorder of autoimmune etiology of unknown cause. Cardiac involvement during systemic lupus erythematosus (SLE) may include the pericardium, myocardium, valvular disease, including Libman-Sacks endocarditis, and coronary arteries. Myocarditis is a severe unusual feature most common in African-American that can be asymptomatic with a prevalence of $8-25 \%$ in patients with systemic lupus erythematosus. Acute myo-pericarditis with subsequent heart failure is a life threatening complication of SLE that needs to get recognized early to prevent mortality to the patient. Steroids is the first line therapy in patients with acute myopericarditis. Here we present a rare case of SLE flare presenting as Acute myopericarditis
\end{abstract}

Volume II Issue I - 202I

\author{
Luis Caraballo, Joel Brooks, Evelyn Bryon, \\ Jose Gascon, Jose G Pantaleon \\ Department of Internal Medicine, Kendall Regional Medical \\ Center, USA
}

\section{Correspondence: Luis Caraballo, Department of internal medicine, Kendall Regional medical center, USA,} Email Icaraball86@gmail.com

Received: December 15, 2020 | Published: February 17, 202

\section{Introduction}

Cardiac involvement during systemic lupus erythematosus (SLE) may include the pericardium, myocardium, valvular disease, including Libman-Sacks endocarditis, and coronary arteries. Symptomatic pericarditis is present in up to $25 \%$ of patients, being the most common cardiac manifestation of lupus. ${ }^{1}$ Myocarditis is a severe unusual feature most common in African-American that can be asymptomatic with a prevalence of $8-25 \%$ in patients with systemic lupus erythematosus. Pericardial effusion can be found in up to $40 \%$ of patients with SLE. Even though uncommon, involvement of the myocardium, pericardium and pericardial effusion (Acute myo-pericarditis) with subsequent heart failure is a life threatening complication of SLE that needs to get recognized early to prevent mortality to the patient.

\section{Case report}

A 22-year-old Afro-Caribbean female was transferred to the hospital for evaluation of new-onset heart failure and pericardial effusion. Her past medical history was significant for Systemic Lupus Erythematosus (SLE) complicated by pericardial effusion at the time of diagnosis two years prior, requiring a pericardial window. Her other medical conditions included rheumatoid arthritis (RA) and medication-induced hypertension.

Two days prior to presentation, the patient had presented to an outside hospital (OSH) for generalized weakness, fatigue, dyspnea and palpitations after discontinuing Imuran due to elevated liver function tests. She underwent computed tomography angiography, which did not show pulmonary embolism, but revealed small bilateral pleural effusion and minimal pericardial effusions, cardiomegaly, adenopathy and hypervascular left hepatic lobe lesion. Transthoracic echocardiography (TTE) report from OSH showed scant pericardial effusion, without evidence of tamponade, EF: $25-30 \%$. Given the patient presentation, she was diagnosed with SLE flare. CK was elevated at 280, myoglobin 7.1, troponin T 0.199, NT Pro BNP 29,638 with a positive ANA, ANA titer 1:1,280, speckled ANA pattern, Anti Ds DNA 518, RNP antibody $>8.0$, Ribosomal RNP 1.4, Sm antibody $>8.0$, SSA antibody $>2.8$, Scleroderma antibody IgG1.4 with a low C4(3) and C3(35). On physical examination. The oral mucosa was dry, the rest of thephysical examination was normal.
The patient was started on 50mg of methylprednisolone IV, hydroxychloroquine $200 \mathrm{mg}$ PO daily and piperacillin- tazobactam $3.375 \mathrm{~g}$ in dextrose $50 \mathrm{~mL}$ IV which resulted in improvement of her symptoms. Patient was discharged with metoprolol succinate $100 \mathrm{mg}$ daily, lisinopril $20 \mathrm{mg}$ daily, prednisone $50 \mathrm{mg}$ daily and follow up with cardiology, rheumatology and cardiac MR in 1 week.

\section{Conclusion}

Systemic lupus erythematosus (SLE) is a chronic, multisystem, inflammatory disorder of autoimmune etiology of unknown cause. Particularly the production of a number of antinuclear antibodies, is a prominent feature of the disease, occurring predominantly in young women. SLE clinical manifestations can vary dramatically from patient to patient. The most common pattern is a mixture of constitutional complaints with skin, musculoskeletal, mild hematologic, and serologic involvement. The pattern that dominates during the first few years of illness tends to prevail throughout the disease course. ${ }^{2-5}$

Cardiac involvement is not uncommon in SLE, evidence of pericardial disease can be found in $80 \%$ of patients followed by $40 \%$ of cases of myocarditis in postmortem examination. ${ }^{4}$ Echocardiographic studies showed global hypokinesia in $6 \%$ of cases in patients with systemic lupus erythematosus. ${ }^{6,7}$ The diagnosis of myocarditis can be supported by laboratory findings(leukocytosis, elevation of inflammatory markers, cardiac enzymes) and transthoracic echocardiography. Electrocardiographic findings such as dysrhythmias or heart block may be seen. Lupus myocarditis and the presence of anti-Ro and antinuclear ribonucleoprotein antibodies should be put in consideration since it has been associated with myocarditis in the past. ${ }^{8}$ Definitive diagnosis can be made by endomyocardial biopsy even though it has limited sensitivity and specificity and histological findings should be taken in the context of the clinical Picture. ${ }^{9,10}$

Acute myo-pericarditis is rare but is needed to recognize early as can present as a life threatening complication of systemic lupus erythematosus. Steroids are the first line treatment of Acute myopericarditis in lupus flare either orally or intravenously. ${ }^{11}$ There is limited information available of long term outcome despite successful initial clinical remission. 


\section{Conflicts of interest}

The author declares no conflicts of interest.

\section{Acknowledgments}

None.

\section{Funding}

None.

\section{References}

1. Dein Eric, Homeyra Douglas, Michelle Petri, et al. Pericarditis in Lupus. Cureus. 2019;11(3):e4166.

2. Karen H Costenbader, David S Pisetsky, Monica Ramirez Curtis. Noncoronary cardiac manifestations of systemic lupus erythematosus in adults. 2021.

3. Prema Bezwada, Ahmed Quadri, Atif Shaikh, et al. Myopericarditis and Pericardial Effusion as the Initial Presentation of Systemic Lupus Erythematosus. Case Rep Med. 2017;2017:6912020.

4. https://www.mdedge.com/ccjm/article/99078/cardiology/when-doespericarditis-merit-workup-autoimmune-or-inflammatory-disease.
5. https://www.uptodate.com/contents/overview-of-theclinical-manifestations-of-systemic-lupus-erythematosus-inadults? search=systemic $\% 201$ upus $\% 20$ erythematosus\&source $=$ search result $\&$ selectedTitle $=1 \sim 150 \&$ usage_type $=$ default $\&$ display_ rank=1\#H16.

6. S Busteed, P Sparrow, C Molloy, et al. Myocarditis as a prognostic indicator in systemic lupus erythematosus. Postgrad Med J. 2 2004;80:366-367.

7. Wijetunga M, Rockson S. Myocarditis in systemic lupus erythematosus. Am J Med. 2002;113(5):419-423.

8. Logar D, Kveder T, Rozman B, et al. Possible association between antiRo antibodies and myocarditis or cardiac conduction defects in adults with systemic lupus erythematosus. Ann Rheum Dis 1990;49(8):627629.

9. Swaak AJ, Huysen V, Smeenk RJ. Antinuclear antibodies in routine analysis: the relevance of putative clinical associations. Ann Rheum Dis. 1993;52(2):110-114.

10. Feldman AM, McNamara D. Myocarditis. $N$ Engl $J$ Med. 2000;343(19):1388-1398.

11. Frustaci A, Gentiloni N, Caldarulo M. Acute myocarditis and left ventricular aneurysmas presentations of systemic lupus erythematosus. Chest. 1996;109(1):282-284. 